\title{
On the Behavior of Solutions of the System of Rational Difference Equations:
}

\author{
$x_{n+1}=x_{n-1} /\left(y_{n} x_{n-1}-1\right), y_{n+1}=y_{n-1} /\left(x_{n} y_{n-1}-1\right)$, \\ and $z_{n+1}=z_{n-1} /\left(y_{n} z_{n-1}-1\right)$
}

\begin{abstract}
Abdullah Selçuk Kurbanli
Department of Mathematics, Faculty of Education, Selcuk University, 42090 Konya, Turkey

Correspondence should be addressed to Abdullah Selçuk Kurbanli, agurban@selcuk.edu.tr

Received 23 December 2010; Accepted 26 January 2011

Academic Editor: Ibrahim Yalcinkaya

Copyright (C) 2011 Abdullah Selçuk Kurbanli. This is an open access article distributed under the Creative Commons Attribution License, which permits unrestricted use, distribution, and reproduction in any medium, provided the original work is properly cited.

We investigate the solutions of the system of difference equations $x_{n+1}=x_{n-1} /\left(y_{n} x_{n-1}-1\right)$, $y_{n+1}=y_{n-1} /\left(x_{n} y_{n-1}-1\right), z_{n+1}=z_{n-1} /\left(y_{n} z_{n-1}-1\right)$, where $y_{0}, y_{-1}, x_{0}, x_{-1}, z_{-1}, z_{0} \in \mathbb{R}$.
\end{abstract}

\section{Introduction}

Recently, there has been great interest in studying difference equation systems. One of the reasons for this is a necessity for some techniques which can be used in investigating equations arising in mathematical models describing real life situations in population biology, economic, probability theory, genetics, psychology, and so forth. There are many papers related to the difference equations system, for example, the following papers.

In [1], Çinar studied the solutions of the systems of the difference equations

$$
x_{n+1}=\frac{1}{y_{n}}, \quad y_{n+1}=\frac{y_{n}}{x_{n-1} y_{n-1}} .
$$

In [2] Papaschinopoulos and Schinas studied the oscillatory behavior, the boundedness of the solutions, and the global asymptotic stability of the positive equilibrium of the system of nonlinear difference equations

$$
x_{n+1}=A+\frac{y_{n}}{x_{n-p}}, \quad y_{n+1}=A+\frac{x_{n}}{y_{n-q}}, \quad n=0,1, \ldots, p, q
$$


In [3] Papaschinopoulos and Schinas proved the boundedness, persistence, the oscillatory behavior, and the asymptotic behavior of the positive solutions of the system of difference equations

$$
x_{n+1}=\sum_{i=0}^{k} \frac{A_{i}}{y_{n-i}^{p_{i}}}, \quad y_{n+1}=\sum_{i=0}^{k} \frac{B_{i}}{x_{n-i}^{q_{i}}} .
$$
equations

In $[4,5]$ Özban studied the positive solutions of the system of rational difference

$$
\begin{array}{cc}
x_{n}=\frac{a}{y_{n-3}}, & y_{n}=\frac{b y_{n-3}}{x_{n-q} y_{n-q}}, \\
x_{n+1}=\frac{1}{y_{n-k}}, & y_{n+1}=\frac{y_{n}}{x_{n-m} y_{n-m-k}} .
\end{array}
$$

In $[6,7]$ Clark and Kulenović investigate the global asymptotic stability

$$
x_{n+1}=\frac{x_{n}}{a+c y_{n}}, \quad y_{n+1}=\frac{y_{n}}{b+d x_{n}} \text {. }
$$

In [8] Camouzis and Papaschinopoulos studied the global asymptotic behavior of positive solutions of the system of rational difference equations

$$
x_{n+1}=1+\frac{x_{n}}{y_{n-m}}, \quad y_{n+1}=1+\frac{y_{n}}{x_{n-m}} .
$$

In [9] Yang et al. considered the behavior of the positive solutions of the system of the difference equations

$$
x_{n}=\frac{a}{y_{n-p}}, \quad y_{n}=\frac{b y_{n-p}}{x_{n-q} y_{n-q}} .
$$

In [10] Kulenović and Nurkanović studied the global asymptotic behavior of solutions of the system of difference equations

$$
x_{n+1}=\frac{a+x_{n}}{b+y_{n}}, \quad y_{n+1}=\frac{c+y_{n}}{d+z_{n}}, \quad z_{n+1}=\frac{e+z_{n}}{f+x_{n}} .
$$

In [11] Zhang et al. investigated the behavior of the positive solutions of the system of difference equations

$$
x_{n}=A+\frac{1}{y_{n-p}}, \quad y_{n}=A+\frac{y_{n-1}}{x_{n-r} y_{n-s}}
$$


In [12] Zhang et al. studied the boundedness, the persistence, and global asymptotic stability of the positive solutions of the system of difference equations

$$
x_{n+1}=A+\frac{y_{n-m}}{x_{n}}, \quad y_{n+1}=A+\frac{x_{n-m}}{y_{n}} .
$$

In [13] Yalcinkaya studied the global asymptotic behavior of a system of two nonlinear difference equations.

In [14] Yalcinkaya et al. investigated the solutions of the system of difference equations

$$
x_{n+1}^{(1)}=\frac{x_{n}^{(2)}}{x_{n}^{(2)}-1}, x_{n+1}^{(2)}=\frac{x_{n}^{(3)}}{x_{n}^{(3)}-1}, \ldots, x_{n+1}^{(k)}=\frac{x_{n}^{(1)}}{x_{n}^{(1)}-1} .
$$

In [15] Yalcinkaya studied the global asymptotic stability of the system of difference equations

$$
z_{n+1}=\frac{t_{n} z_{n-1}+a}{t_{n}+z_{n-1}}, \quad t_{n+1}=\frac{z_{n} t_{n-1}+a}{z_{n}+t_{n-1}}
$$

In [16] Irićanin and Stević studied the positive solutions of the system of difference equations

$$
\begin{gathered}
x_{n+1}^{(1)}=\frac{1+x_{n}^{(2)}}{x_{n-1}^{(3)}}, x_{n+1}^{(2)}=\frac{1+x_{n}^{(3)}}{x_{n-1}^{(4)}}, \ldots, x_{n+1}^{(k)}=\frac{1+x_{n}^{(1)}}{x_{n-1}^{(2)}}, \\
x_{n+1}^{(1)}=\frac{1+x_{n}^{(2)}+x_{n-1}^{(3)}}{x_{n-2}^{(4)}}, x_{n+1}^{(2)}=\frac{1+x_{n}^{(3)}+x_{n-1}^{(4)}}{x_{n-2}^{(5)}}, \ldots, x_{n+1}^{(k)}=\frac{1+x_{n}^{(1)}+x_{n-1}^{(2)}}{x_{n-2}^{(3)}} .
\end{gathered}
$$

In [17] Kurbanli et al. studied the behavaior of positive solutions of the system of rational difference equations

$$
x_{n+1}=\frac{x_{n-1}}{y_{n} x_{n-1}+1}, \quad y_{n+1}=\frac{y_{n-1}}{x_{n} y_{n-1}+1} .
$$

Also see references. system

In this paper, we investigate the behavior of the solutions of the difference equations

$$
x_{n+1}=\frac{x_{n-1}}{y_{n} x_{n-1}-1}, \quad y_{n+1}=\frac{y_{n-1}}{x_{n} y_{n-1}-1}, \quad z_{n+1}=\frac{z_{n-1}}{y_{n} z_{n-1}-1}
$$

where the initial conditions are arbitrary real numbers. 
Theorem 1.1. Let $y_{0}, y_{-1}, x_{0}, x_{-1}, z_{-1}, z_{0} \in \mathbb{R}$ be arbitrary real numbers and $y_{0}=a, y_{-1}=b, x_{0}=$ $c, x_{-1}=d, z_{0}=e, z_{-1}=f$ and let $\left\{x_{n}, y_{n}, z_{n}\right\}$ be a solution of the system (1.15). Also, assume that $a f \neq 1$ and $b c \neq 1$ then all solutions of (1.15) are

$$
\begin{aligned}
& x_{n}= \begin{cases}\frac{d}{(a d-1)^{n}}, & n \text {-odd, } \\
c(b c-1)^{n}, & n \text {-even, }\end{cases} \\
& y_{n}= \begin{cases}\frac{b}{(b c-1)^{n}}, & n \text {-odd, } \\
a(a d-1)^{n}, & n \text {-even, }\end{cases} \\
& z_{n}=\left\{\begin{array}{c}
\frac{f}{(-1)^{0}\left(\begin{array}{c}
n \\
0
\end{array}\right) a^{n} f d^{n-1}+(-1)^{1}\left(\begin{array}{c}
n \\
1
\end{array}\right) a^{n-1} f d^{n-2}+\cdots+(-1)^{n-1}\left(\begin{array}{c}
n \\
n-1
\end{array}\right) a^{1} f d^{0}+(-1)^{n}\left(\begin{array}{c}
n \\
n
\end{array}\right)} \\
n \text {-odd } \\
(-1)^{n} \frac{(b c-1)^{n} e}{(-1)^{n}\left(\begin{array}{c}
n \\
1
\end{array}\right) b^{1} c^{0} e+\cdots+(-1)^{1}\left(\begin{array}{c}
n \\
n
\end{array}\right) b^{n} c^{n-1} e+(-1)^{0}\left(\begin{array}{c}
n \\
0
\end{array}\right) b^{n} c^{n}+\cdots+(-1)^{n}\left(\begin{array}{c}
n \\
n
\end{array}\right) b^{0} c^{0}} \\
n \text {-even. }
\end{array},\right.
\end{aligned}
$$

Proof. For $n=0,1,2,3$, we have

$$
\begin{gathered}
x_{1}=\frac{x_{-1}}{y_{0} x_{-1}-1}=\frac{d}{a d-1}, \\
y_{1}=\frac{y_{-1}}{x_{0} y_{-1}-1}=\frac{b}{b c-1}, \\
z_{1}=\frac{z_{-1}}{y_{0} z_{-1}-1}=\frac{f}{a f-1}, \\
x_{2}=\frac{x_{0}}{y_{1} x_{0}-1}=\frac{c}{(b /(b c-1)) c-1}=\frac{c(b c-1)}{b c-b c+1}=c(b c-1), \\
y_{2}=\frac{y_{0}}{x_{1} y_{0}-1}=\frac{a}{(d /(a d-1)) a-1}=\frac{a(a d-1)}{a d-a d+1}=a(a d-1), \\
z_{2}=\frac{z_{0}}{y_{1} z_{0}-1}=\frac{e}{(b /(b c-1)) e-1}=\frac{e(b c-1)}{b e-b c+1}=-\frac{e(b c-1)}{-1 \cdot b e+b c-1}, \\
y_{3}=\frac{x_{3}=\frac{x_{1}}{y_{2} x_{1}-1}=\frac{d /(a d-1)}{a(a d-1) \cdot d /(a d-1)-1}=\frac{d}{(a d-1)^{2}},}{x_{2} y_{1}-1}=\frac{b /(b c-1)}{c(b c-1) \cdot b /(b c-1)-1}=\frac{b /(b c-1)}{b c-1}=\frac{b}{(b c-1)^{2}}, \\
z_{3}=\frac{f /(a f-1)}{y_{2} z_{1}-1}=\frac{f /(a f-1)}{a(a d-1) \cdot f /(a f-1)-1}=\frac{f\left(a^{2} d f-a f-a f+1\right) /(a f-1)}{a^{2} d f-2 a f+1}
\end{gathered}
$$


for $n=k$ assume that

$$
\begin{aligned}
& x_{k}= \begin{cases}\frac{d}{(a d-1)^{k}}, & k \text {-odd } \\
c(b c-1)^{k}, & k \text {-even }\end{cases} \\
& y_{k}= \begin{cases}\frac{b}{(b c-1)^{k}}, & k \text {-odd, } \\
a(a d-1)^{k}, & k \text {-even, }\end{cases} \\
& z_{2 k-1}=\frac{f}{(-1)^{0}\left(\begin{array}{c}
k \\
0
\end{array}\right) a^{k} f d^{k-1}+(-1)^{1}\left(\begin{array}{c}
k \\
1
\end{array}\right) a^{k-1} f d^{k-2}+\cdots+(-1)^{k-1}\left(\begin{array}{c}
k \\
k-1
\end{array}\right) a^{1} f d^{0}+(-1)^{k}\left(\begin{array}{c}
k \\
k
\end{array}\right)} \\
& k=1,2, \ldots, \\
& z_{2 k}=(-1)^{k} \frac{(b c-1)^{k} e}{(-1)^{k}\left(\begin{array}{c}
k \\
1
\end{array}\right) b^{1} c^{0} e+\cdots+(-1)^{1}\left(\begin{array}{l}
k \\
k
\end{array}\right) b^{k} c^{k-1} e+(-1)^{0}\left(\begin{array}{l}
k \\
0
\end{array}\right) b^{k} c^{k}+\cdots+(-1)^{k}\left(\begin{array}{l}
k \\
k
\end{array}\right) b^{1} c^{1}}, \\
& k=1,2, \ldots
\end{aligned}
$$

are true. Then for $n=k+1$ we will show that (1.16) is true. From (1.15), we have

$$
x_{2 k+1}=\frac{x_{2 k-1}}{y_{2 k} x_{2 k-1}-1}=\frac{d /(a d-1)^{k}}{a(a d-1)^{k} \cdot d /(a d-1)^{k}-1}=\frac{d}{(a d-1)^{k+1}} .
$$

Also, similarly from (1.15), we have

$$
\begin{gathered}
y_{2 k+1}=\frac{y_{2 k-1}}{x_{2 k} y_{2 k-1}-1} \frac{b /(b c-1)^{k}}{c(b c-1)^{k} \cdot b /(b c-1)^{k}-1}=\frac{b}{(b c-1)^{k+1}}, \\
\left(\begin{array}{l}
n \\
k
\end{array}\right)=\frac{n !}{(n-k) ! \cdot k !^{\prime}} \\
\left(\begin{array}{l}
n \\
k
\end{array}\right)+\left(\begin{array}{c}
n \\
k-1
\end{array}\right)=\left(\begin{array}{c}
n+1 \\
k
\end{array}\right) \\
\left(\begin{array}{l}
n \\
0
\end{array}\right)=\left(\begin{array}{l}
n \\
n
\end{array}\right)=\left(\begin{array}{c}
n+1 \\
n+1
\end{array}\right)
\end{gathered}
$$


from properties of Binomial coefficients,

$$
\begin{aligned}
z_{2 k+1} & =\frac{z_{2 k-1}}{y_{2 k} z_{2 k-1}-1} \\
& =\frac{f /\left((-1)^{0}\left(\begin{array}{c}
k \\
0
\end{array}\right) a^{k} f d^{k-1}+\cdots+(-1)^{k-1}\left(\begin{array}{c}
k \\
k-1
\end{array}\right) a^{1} f d^{0}+(-1)^{k}\left(\begin{array}{c}
k \\
k
\end{array}\right)\right)}{a(a d-1)^{k} f /\left((-1)^{0}\left(\begin{array}{c}
k \\
0
\end{array}\right) a^{k} f d^{k-1}+\cdots+(-1)^{k-1}\left(\begin{array}{c}
k \\
k-1
\end{array}\right) a^{1} f d^{0}+(-1)^{k}\left(\begin{array}{c}
k \\
k
\end{array}\right)\right)-1} \\
& =\frac{f}{(-1)^{0}\left(\begin{array}{c}
k+1 \\
0
\end{array}\right) a^{k+1} f d^{k}+(-1)^{1}\left(\begin{array}{c}
k+1 \\
1
\end{array}\right) a^{k} f d^{k-1}+\cdots+(-1)^{k}\left(\begin{array}{c}
k+1 \\
k
\end{array}\right) a^{1} f d^{0}+(-1)^{k+!}\left(\begin{array}{c}
k+1 \\
k+1
\end{array}\right)}
\end{aligned}
$$

written and accurate.

Also, we have

$$
\begin{aligned}
& x_{2 k+2}=\frac{x_{2 k}}{y_{2 k+1} x_{2 k}-1}=\frac{c(b c-1)^{k}}{\left(b /(b c-1)^{k+1}\right) c(b c-1)^{k}-1}=\frac{c(b c-1)^{k}}{b c /(b c-1)-1}=c(b c-1)^{k+1}, \\
& y_{2 k+2}=\frac{y_{2 k}}{x_{2 k+1} y_{2 k}-1}=\frac{a(a d-1)^{k}}{\left(d /(a d-1)^{k+1}\right) \cdot a(a d-1)^{k}-1}=\frac{a(a d-1)^{k}}{a d /(a d-1)-1}=a(a d-1)^{k+!}, \\
& z_{2 k+2}=\frac{z_{2 k}}{y_{2 k+1} z_{2 k}-1} \\
& =\frac{(-1)^{k}(b c-1)^{k} e /\left(A+(-1)^{0}\left(\begin{array}{c}
k \\
0
\end{array}\right) b^{k} c^{k}+\cdots+(-1)^{k}\left(\begin{array}{c}
k \\
k
\end{array}\right) b^{0} c^{0}\right)}{\left(b /(b c-1)^{k+1}\right) \cdot(-1)^{k}(b c-1)^{k} e /\left(A+(-1)^{0}\left(\begin{array}{c}
k \\
0
\end{array}\right) b^{k} c^{k}+\cdots+(-1)^{k}\left(\begin{array}{c}
k \\
k
\end{array}\right) b^{0} c^{0}\right)-1}=(-1)^{k+1} \\
& \quad \times \frac{(b c-1)^{k+1} e}{(-1)^{k+1}\left(\begin{array}{c}
k+1 \\
1
\end{array}\right) b^{1} c^{0} e+\cdots+(-1)^{1}\left(\begin{array}{c}
k+1 \\
k+1
\end{array}\right) b^{k+1} c^{k} e+(-1)^{0}\left(\begin{array}{c}
k+1 \\
0
\end{array}\right) b^{k+1} c^{k+1}+\cdots+(-1)^{k+1}\left(\begin{array}{c}
k+1 \\
k+1
\end{array}\right) b^{1} c^{1}}, \\
& (1.22)
\end{aligned}
$$

where $\mathcal{A}$ denotes $(-1)^{k}\left(\begin{array}{c}k \\ 1\end{array}\right) b^{1} c^{0} e+\cdots+(-1)^{1}\left(\begin{array}{c}k \\ k\end{array}\right) b^{k} c^{k-1} e$.

Corollary 1.2. Let $a, b, c, d$ be only positive real numbers or negative real numbers and let $e, f$ be arbitrary nonnegative real numbers, and let $\left\{x_{n}, y_{n}, z_{n}\right\}$ be a solution of the system (1.15). If $a d \neq 1, b c \neq 1,0<a d<1$ and $0<b c<1$ then one has

$$
\begin{gathered}
\lim _{n \rightarrow \infty} x_{2 n-1}=\lim _{n \rightarrow \infty} y_{2 n-1}=\infty, \\
\lim _{n \rightarrow \infty} x_{2 n}=\lim _{n \rightarrow \infty} y_{2 n}=0 .
\end{gathered}
$$


Proof. From $a d \neq 1, b c \neq 1,0<a d<1$ and $0<b c<1$ we have $-1<a d-1<0$ and $-1<$ $b c-1<0$. Hence, we have

$$
\begin{array}{ll}
x_{2 n-1}=\frac{d}{(a d-1)^{n}}, & y_{2 n-1}=\frac{b}{(b c-1)^{n}}, \\
x_{2 n}=c(b c-1)^{n}, & y_{2 n}=a(a d-1)^{n} .
\end{array}
$$

Then,

$$
\begin{gathered}
\lim _{n \rightarrow \infty} x_{2 n-1}=\lim _{n \rightarrow \infty} \frac{d}{(a d-1)^{n}}= \begin{cases}+\infty, & d>0, n \text {-even } \\
+\infty, & d<0, n \text {-odd } \\
-\infty, & d>0, n \text {-odd } \\
-\infty, & d<0, n \text {-even, }\end{cases} \\
\lim _{n \rightarrow \infty} y_{2 n-1}=\lim _{n \rightarrow \infty} \frac{b}{(b c-1)^{n}}= \begin{cases}+\infty, & b>0, n \text {-even } \\
+\infty, & b<0, n \text {-odd } \\
-\infty, & b>0, n \text {-odd } \\
-\infty, & b<0, n \text {-even. }\end{cases}
\end{gathered}
$$

Also,

$$
\begin{aligned}
& \lim _{n \rightarrow \infty} x_{2 n}=\lim _{n \rightarrow \infty} c(b c-1)^{n}=0, \\
& \lim _{n \rightarrow \infty} y_{2 n}=\lim _{n \rightarrow \infty} a(a d-1)^{n}=0 .
\end{aligned}
$$

Example 1.3. If $y(0)=-1 / 2, y(-1)=-1 / 2, x(0)=-1 / 2, x(-1)=-1 / 2$, then the solutions of (1.15) can be represented by Table 1.

Corollary 1.4. Let $a, b, c, d$ be only positive real numbers or negative real numbers and let $e, f$ be arbitrary nonnegative real numbers, and let $\left\{x_{n}, y_{n}, z_{n}\right\}$ be a solution of the system (1.15). If $a d \neq 1, b c \neq 1,1<a d<2$ and $1<b c<2$ then one has

$$
\begin{gathered}
\lim _{n \rightarrow \infty} x_{2 n-1}=\lim _{n \rightarrow \infty} y_{2 n-1}=\infty, \\
\lim _{n \rightarrow \infty} x_{2 n}=\lim _{n \rightarrow \infty} y_{2 n}=0 .
\end{gathered}
$$


Table 1

\begin{tabular}{lcccccccc}
\hline$i$ & 1 & 2 & 3 & 4 & 5 & 6 & 7 & 8 \\
\hline$x_{2 i-1}$ & 0.6667 & -0.8889 & 1.1852 & -1.5803 & 2.1070 & -2.8093 & 3.7458 & -4.9944 \\
$x_{2 i}$ & 0.3750 & -0.2813 & 0.2109 & -0.1582 & 0.1187 & -0.0890 & 0.0667 & -0.0501 \\
$y_{2 i-1}$ & 0.6667 & -0.8889 & 1.1852 & -1.5803 & 2.1070 & -2.8093 & 3.7458 & -4.9944 \\
$y_{2 i}$ & 0.3750 & -0.2813 & 0.2109 & -0.1582 & 0.1187 & -0.0890 & 0.0667 & -0.0501 \\
$z_{2 i-1}$ & 0.6667 & -0.8889 & 1.1852 & -1.5803 & 2.1070 & -2.8093 & 3.7458 & -4.9944 \\
$z_{2 i}$ & 0.3750 & -0.2813 & 0.2109 & -0.1582 & 0.1187 & -0.0890 & 0.0667 & -0.0501 \\
\hline
\end{tabular}

Proof. From $a d \neq 1, b c \neq 1,1<a d<2$ and $1<b c<2$ we have $0<a d-1<1$ and $0<b c-1<1$. Hence, we have

$$
\begin{array}{ll}
x_{2 n-1}=\frac{d}{(a d-1)^{n}}, & y_{2 n-1}=\frac{b}{(b c-1)^{n}}, \\
x_{2 n}=c(b c-1)^{n}, & y_{2 n}=a(a d-1)^{n} .
\end{array}
$$

Then,

$$
\begin{aligned}
& \lim _{n \rightarrow \infty} x_{2 n-1}=\lim _{n \rightarrow \infty} \frac{d}{(a d-1)^{n}}= \begin{cases}+\infty, & d>0 \\
-\infty, & d<0,\end{cases} \\
& \lim _{n \rightarrow \infty} y_{2 n-1}=\lim _{n \rightarrow \infty} \frac{b}{(b c-1)^{n}}= \begin{cases}+\infty, & b>0 \\
-\infty, & b<0 .\end{cases}
\end{aligned}
$$

Also,

$$
\begin{aligned}
& \lim _{n \rightarrow \infty} x_{2 n}=\lim _{n \rightarrow \infty} c(b c-1)^{n}=0, \\
& \lim _{n \rightarrow \infty} y_{2 n}=\lim _{n \rightarrow \infty} a(a d-1)^{n}=0 .
\end{aligned}
$$

Example 1.5. If $y(0)=3 / 2, y(-1)=3 / 4, x(0)=3 / 2, x(-1)=3 / 4, z(0)=3 / 2, z(-1)=3 / 4$ then the solutions of (1.15) can be represented by Table 2 .

Corollary 1.6. Let $a, b, c, d$ be only positive real numbers or negative real numbers and let $e, f$ be arbitrary nonnegative real numbers, and let $\left\{x_{n}, y_{n}, z_{n}\right\}$ be a solution of the system (1.15). 
If $-\infty<a d<0$ and $-\infty<b c<0$ then one has

$$
\begin{gathered}
\lim _{n \rightarrow \infty} x_{2 n-1}=\lim _{n \rightarrow \infty} y_{2 n-1}=0, \\
\lim _{n \rightarrow \infty} x_{2 n}=\lim _{n \rightarrow \infty} y_{2 n}=\infty .
\end{gathered}
$$

Proof. From $-\infty<a d<0$ and $-\infty<b c<0$ we have $-\infty<a d-1<-1$ and $-\infty<b c-1<-1$. Hence, we have

$$
\begin{array}{cl}
x_{2 n-1}=\frac{d}{(a d-1)^{n}}, & y_{2 n-1}=\frac{b}{(b c-1)^{n}}, \\
x_{2 n}=c(b c-1)^{n}, & y_{2 n}=a(a d-1)^{n} .
\end{array}
$$

Then,

$$
\begin{aligned}
& \lim _{n \rightarrow \infty} x_{2 n-1}=\lim _{n \rightarrow \infty} \frac{d}{(a d-1)^{n}}=0 \\
& \lim _{n \rightarrow \infty} y_{2 n-1}=\lim _{n \rightarrow \infty} \frac{b}{(b c-1)^{n}}=0 .
\end{aligned}
$$

Also,

$$
\begin{gathered}
\lim _{n \rightarrow \infty} x_{2 n}=\lim _{n \rightarrow \infty} c(b c-1)^{n}= \begin{cases}+\infty, & c>0, n \text {-even } \\
+\infty, & c<0, n \text {-odd } \\
-\infty, & c>0, n \text {-odd } \\
-\infty, & c<0, n \text {-even }\end{cases} \\
\lim _{n \rightarrow \infty} y_{2 n}=\lim _{n \rightarrow \infty} a(a d-1)^{n}= \begin{cases}+\infty, & a>0, n \text {-even } \\
+\infty, & a<0, n \text {-odd } \\
-\infty, & a>0, n \text {-odd } \\
-\infty, & a<0, n \text {-even }\end{cases}
\end{gathered}
$$

Example 1.7. If $y(0)=-3 / 2, y(-1)=-3 / 4, x(0)=3 / 2, x(-1)=3 / 4, z(0)=3 / 2, z(-1)=$ $3 / 4$ then the solutions of (1.15) can be represented by Table 3 . 
Table 2

\begin{tabular}{lcccccccc}
\hline$i$ & 1 & 2 & 3 & 4 & 5 & 6 & 7 & 8 \\
\hline$x_{2 i-1}$ & 6 & 48 & 384 & 3072 & 24576 & $1.967 \cdot 10^{5}$ & $1.573 \cdot 10^{6}$ & $1.258 \cdot 10^{7}$ \\
$x_{2 i}$ & 0.188 & 0.023 & 0.003 & 0.0004 & 0.00004 & 0.00001 & $7.153 \cdot 10^{-7}$ & $8.941 \cdot 10^{-8}$ \\
$y_{2 i-1}$ & 6 & 48 & 384 & 3072 & 24576 & $1.967 \cdot 10^{5}$ & $1.573 \cdot 10^{6}$ & $1.258 \cdot 10^{7}$ \\
$y_{2 i}$ & 0.188 & 0.023 & 0.003 & 0.0004 & 0.00004 & 0.00001 & $7.153 \cdot 10^{-7}$ & $8.941 \cdot 10^{-8}$ \\
$z_{2 i-1}$ & 6 & 48 & 384 & 3072 & 24576 & $1.967 \cdot 10^{5}$ & $1.573 \cdot 10^{6}$ & $1.258 \cdot 10^{7}$ \\
$z_{2 i}$ & 0.188 & 0.023 & 0.003 & 0.0004 & 0.00004 & 0.00001 & $7.153 \cdot 10^{-7}$ & $8.941 \cdot 10^{-8}$ \\
\hline
\end{tabular}

Table 3

\begin{tabular}{lcccccccc}
\hline$i$ & 1 & 2 & 3 & 4 & 5 & 6 & 7 & 8 \\
\hline$x_{2 i-1}$ & -0.3529 & 0.1661 & -0.0782 & 0.0368 & -0.0173 & 0.0081 & -0.0038 & 0.0018 \\
$x_{2 i}$ & -3.1875 & 6.7734 & -14.3936 & 30.5863 & -64.9959 & 138.1163 & -293.4971 & 623.6813 \\
$y_{2 i-1}$ & 0.3529 & -0.1661 & 0.0782 & -0.0368 & 0.0173 & -0.0081 & 0.0038 & -0.0018 \\
$y_{2 i}$ & 3.1875 & -6.7734 & 14.3936 & -30.5863 & 64.9959 & -138.1163 & 293.4971 & -623.6813 \\
$z_{2 i-1}$ & -0.3529 & 0.1661 & -0.0782 & 0.0368 & -0.0173 & 0.0081 & -0.0038 & 0.0018 \\
$z_{2 i}$ & -3.1875 & 6.7734 & -14.3936 & 30.5863 & -64.9959 & 138.1163 & -293.4971 & 623.6813 \\
\hline
\end{tabular}

Corollary 1.8. Let $a, b, c, d$ be only positive real numbers or negative real numbers and let $e, f$ be arbitrary nonnegative real numbers, and let $\left\{x_{n}, y_{n}, z_{n}\right\}$ be a solution of the system (1.15). If $2<$ $a d<+\infty$ and $2<b c<+\infty$ then one has

$$
\begin{gathered}
\lim _{n \rightarrow \infty} x_{2 n-1}=\lim _{n \rightarrow \infty} y_{2 n-1}=0, \\
\lim _{n \rightarrow \infty} x_{2 n}=\lim _{n \rightarrow \infty} y_{2 n}=\infty .
\end{gathered}
$$

Proof. From $2<a d<+\infty$ and $2<b c<+\infty$ we have $1<a d-1<+\infty$ and $1<b c-1<+\infty$. Hence, we have

$$
\begin{array}{cl}
x_{2 n-1}=\frac{d}{(a d-1)^{n}}, & y_{2 n-1}=\frac{b}{(b c-1)^{n}}, \\
x_{2 n}=c(b c-1)^{n}, & y_{2 n}=a(a d-1)^{n} .
\end{array}
$$

Then,

$$
\begin{aligned}
& \lim _{n \rightarrow \infty} x_{2 n-1}=\lim _{n \rightarrow \infty} \frac{d}{(a d-1)^{n}}=0, \\
& \lim _{n \rightarrow \infty} y_{2 n-1}=\lim _{n \rightarrow \infty} \frac{b}{(b c-1)^{n}}=0 .
\end{aligned}
$$


Table 4

\begin{tabular}{lcccccccc}
\hline$i$ & 1 & 2 & 3 & 4 & 5 & 6 & 7 & 8 \\
\hline$x_{2 i-1}$ & 0.546 & 0.198 & 0.072 & 0.026 & 0.010 & 0.004 & 0.001 & 0.0005 \\
$x_{2 i}$ & 4.125 & 11.344 & 31.195 & 85.787 & 235.915 & 648.765 & 1784.104 & 4906.285 \\
$y_{2 i-1}$ & 0.909 & 0.331 & 0.120 & 0.044 & 0.016 & 0.006 & 0.002 & 0.001 \\
$y_{2 i}$ & 6.875 & 18.906 & 51.992 & 142.979 & 393.191 & 1081.275 & 2973.506 & 8177.142 \\
$z_{2 i-1}$ & 0.546 & 0.198 & 0.072 & 0.026 & 0.010 & 0.004 & 0.001 & 0.0005 \\
$z_{2 i}$ & 4.125 & 11.344 & 31.195 & 85.787 & 235.915 & 648.765 & 1784.104 & 4906.285 \\
\hline
\end{tabular}

Also,

$$
\begin{aligned}
& \lim _{n \rightarrow \infty} x_{2 n}=\lim _{n \rightarrow \infty} c(b c-1)^{n}= \begin{cases}+\infty, & c>0 \\
-\infty, & c<0,\end{cases} \\
& \lim _{n \rightarrow \infty} y_{2 n}=\lim _{n \rightarrow \infty} a(a d-1)^{n}= \begin{cases}+\infty, & a>0 \\
-\infty, & a<0 .\end{cases}
\end{aligned}
$$

Example 1.9. If $y(0)=5 / 2, y(-1)=5 / 2, x(0)=3 / 2, x(-1)=3 / 2, z(0)=3 / 2, z(-1)=3 / 2$ then the solutions of (1.15) can be represented by Table 4 .

\section{Acknowledgment}

The authors are grateful to the anonymous referees for their valuable suggestions that improved the quality of this study.

\section{References}

[1] C. Çinar, "On the positive solutions of the difference equation system $x_{n+1}=1 / y_{n}, y_{n+1}=$ $y_{n} / x_{n-1} y_{n-1}, "$ Applied Mathematics and Computation, vol. 158, no. 2, pp. 303-305, 2004.

[2] G. Papaschinopoulos and C. J. Schinas, "On a system of two nonlinear difference equations," Journal of Mathematical Analysis and Applications, vol. 219, no. 2, pp. 415-426, 1998.

[3] G. Papaschinopoulos and C. J. Schinas, "On the system of two difference equations," Journal of Mathematical Analysis and Applications, vol. 273, no. 2, pp. 294-309, 2002.

[4] A. Y. Özban, "On the system of rational difference equations $x_{n}=a / y_{n-3}, y_{n}=b y_{n-3} / x_{n-q} y_{n-q}$," Applied Mathematics and Computation, vol. 188, no. 1, pp. 833-837, 2007.

[5] A. Y. Özban, "On the positive solutions of the system of rational difference equations $x_{n+1}=1 / y_{n-k}$, $y_{n+1}=y_{n} / x_{n-m} y_{n-m-k}$," Journal of Mathematical Analysis and Applications, vol. 323, no. 1, pp. 26-32, 2006.

[6] D. Clark and M. R. S. Kulenović, "A coupled system of rational difference equations," Computers $\mathcal{E}$ Mathematics with Applications, vol. 43, no. 6-7, pp. 849-867, 2002.

[7] D. Clark, M. R. S. Kulenović, and J. F. Selgrade, "Global asymptotic behavior of a two-dimensional difference equation modelling competition," Nonlinear Analysis. Theory, Methods \& Applications, vol. 52, no. 7, pp. 1765-1776, 2003.

[8] E. Camouzis and G. Papaschinopoulos, "Global asymptotic behavior of positive solutions on the system of rational difference equations $x_{n+1}=1+x_{n} / y_{n-m}, y_{n+1}=1+y_{n} / x_{n-m}, "$ Applied Mathematics Letters, vol. 17, no. 6, pp. 733-737, 2004. 
[9] X. Yang, Y. Liu, and S. Bai, "On the system of high order rational difference equations $x_{n}=$ $a / y_{n-p}, y_{n}=b y_{n-p} / x_{n-q} y_{n-q}, "$ Applied Mathematics and Computation, vol. 171, no. 2, pp. 853-856, 2005.

[10] M. R. S. Kulenović and Z. Nurkanović, "Global behavior of a three-dimensional linear fractional system of difference equations," Journal of Mathematical Analysis and Applications, vol. 310, no. 2, pp. 673-689, 2005.

[11] Y. Zhang, X. Yang, G. M. Megson, and D. J. Evans, “On the system of rational difference equations $x_{n}=A+1 / y_{n-p}, y_{n}=A+y_{n-1} / x_{n-r} y_{n-s}, "$ Applied Mathematics and Computation, vol. 176, no. 2, pp. 403-408, 2006.

[12] Y. Zhang, X. Yang, D. J. Evans, and C. Zhu, "On the nonlinear difference equation system $x_{n+1}=$ $A+y_{n-m} / x_{n}, y_{n+1}=A+x_{n-m} / y_{n}$," Computers $\mathcal{E}$ Mathematics with Applications, vol. 53, no. 10, pp. 1561-1566, 2007.

[13] I. Yalcinkaya, "On the global asymptotic behavior of a system of two nonlinear difference equations," Ars Combinatoria, vol. 95, pp. 151-159, 2010.

[14] I. Yalcinkaya, C. Çinar, and M. Atalay, "On the solutions of systems of difference equations," Advances in Difference Equations, vol. 2008, Article ID 143943, 9 pages, 2008.

[15] I. Yalcinkaya, "On the global asymptotic stability of a second-order system of difference equations," Discrete Dynamics in Nature and Society, vol. 2008, Article ID 860152, 12 pages, 2008.

[16] B. D. Irićanin and S. Stević, "Some systems of nonlinear difference equations of higher order with periodic solutions," Dynamics of Continuous, Discrete $\mathcal{E}$ Impulsive Systems. Series A. Mathematical Analysis, vol. 13, no. 3-4, pp. 499-507, 2006.

[17] A. S. Kurbanl1, C. Çinar, and I. Yalcinkaya, "On the behavaior of positive solutions of the system of rational difference equations $x_{n+1}=x_{n-1} /\left(y_{n} x_{n-1}\right)+1, y_{n+1}=y_{n-1} /\left(x_{n} y_{n-1}\right)+1$, , Mathematical and Computer Modelling, vol. 53, no. 5-6, pp. 1261-1267, 2011. 


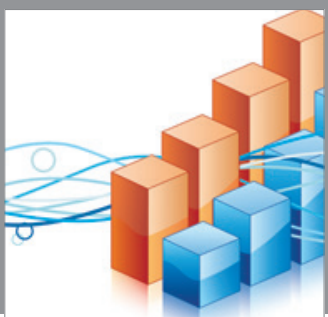

Advances in

Operations Research

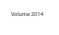

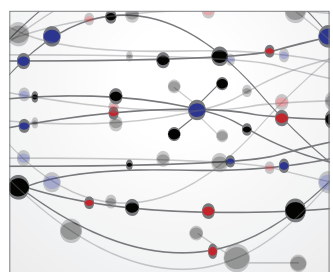

\section{The Scientific} World Journal
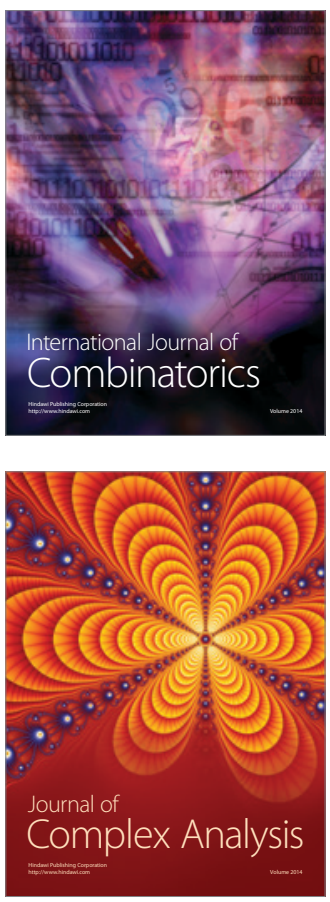

International Journal of

Mathematics and

Mathematical

Sciences
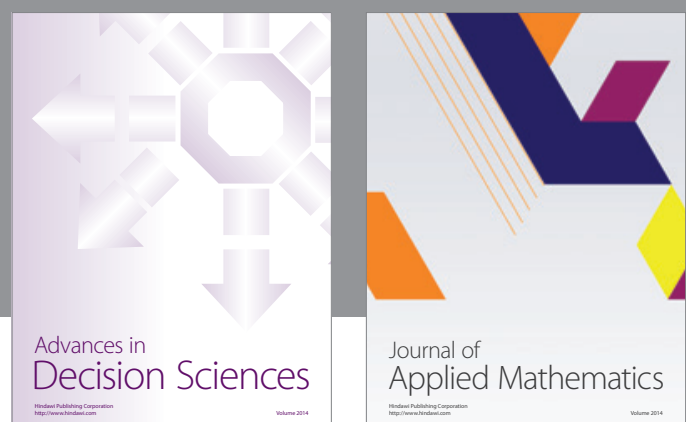

Journal of

Applied Mathematics
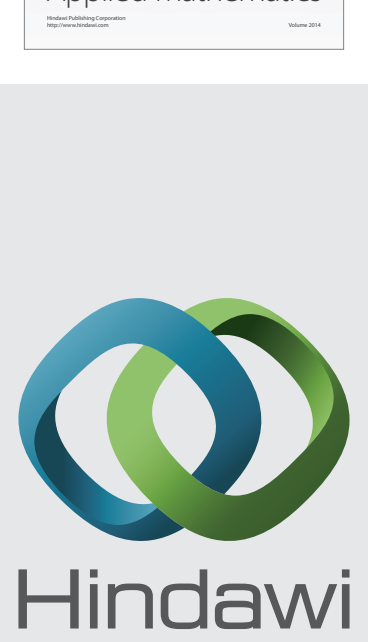

Submit your manuscripts at http://www.hindawi.com
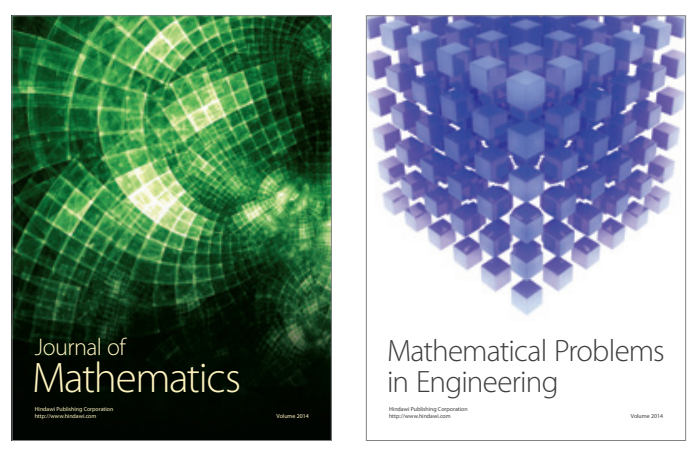

Mathematical Problems in Engineering
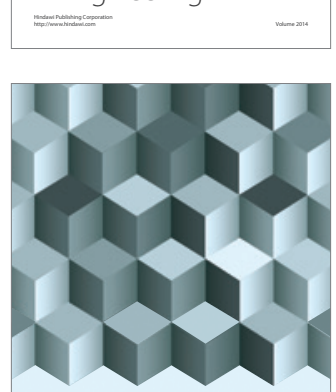

Journal of

Function Spaces
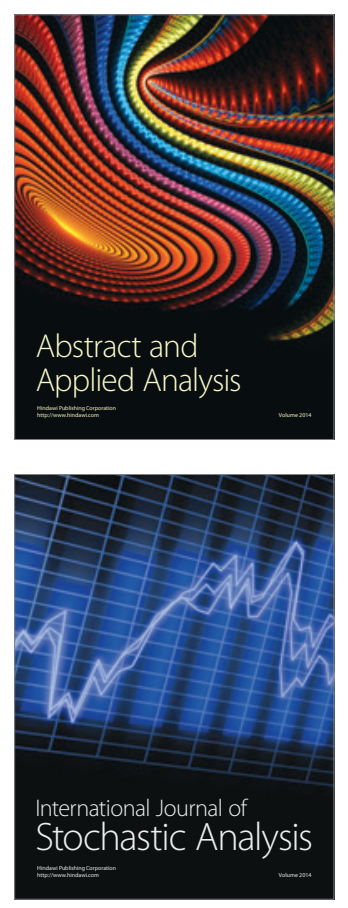

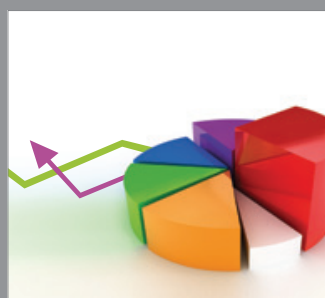

ournal of

Probability and Statistics

Promensencen
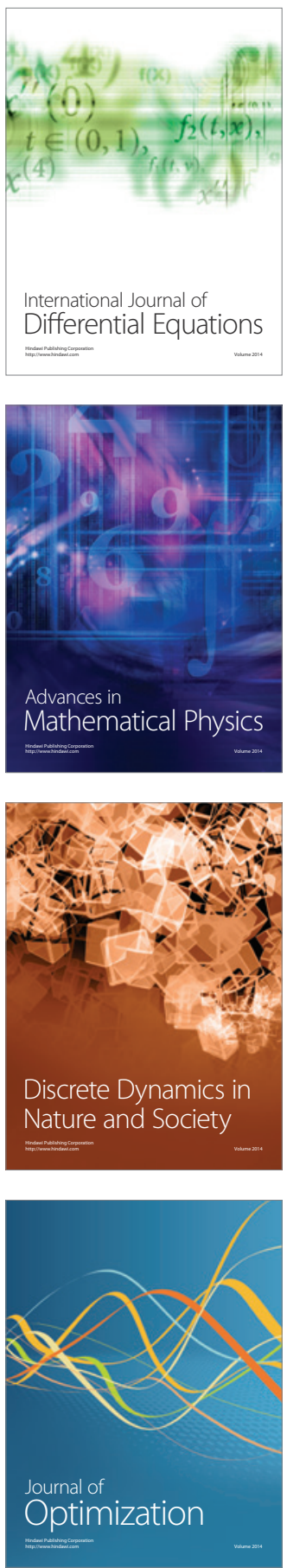